\title{
TU/e EnNHOUN

\section{Prediction of anisotropic crystal-melt interfacial free energy of sugar alcohols through molecular simulations}

\section{Citation for published version (APA):}

Zhang, H., Nedea, S. V., Rindt, C. C. M., Zondag, H. A., \& Smeulders, D. M. J. (2014). Prediction of anisotropic crystal-melt interfacial free energy of sugar alcohols through molecular simulations. In Proceedings of the 15th International Heat Transfer Conference (IHTC-15), August 10-15, 2014, Kyoto, Japan (pp. IHTC15-8636-). Begell House Inc..

Document status and date:

Published: 01/01/2014

\section{Document Version:}

Publisher's PDF, also known as Version of Record (includes final page, issue and volume numbers)

\section{Please check the document version of this publication:}

- A submitted manuscript is the version of the article upon submission and before peer-review. There can be important differences between the submitted version and the official published version of record. People interested in the research are advised to contact the author for the final version of the publication, or visit the $\mathrm{DOI}$ to the publisher's website.

- The final author version and the galley proof are versions of the publication after peer review.

- The final published version features the final layout of the paper including the volume, issue and page numbers.

Link to publication

\section{General rights}

Copyright and moral rights for the publications made accessible in the public portal are retained by the authors and/or other copyright owners and it is a condition of accessing publications that users recognise and abide by the legal requirements associated with these rights.

- Users may download and print one copy of any publication from the public portal for the purpose of private study or research.

- You may not further distribute the material or use it for any profit-making activity or commercial gain

- You may freely distribute the URL identifying the publication in the public portal.

If the publication is distributed under the terms of Article 25fa of the Dutch Copyright Act, indicated by the "Taverne" license above, please follow below link for the End User Agreement:

www.tue.nl/taverne

Take down policy

If you believe that this document breaches copyright please contact us at:

openaccess@tue.nl

providing details and we will investigate your claim. 


\title{
PREDICTION OF ANISOTROPIC CRYSTAL-MELT INTERFACIAL FREE ENERGY OF SUGAR ALCOHOLS THROUGH MOLECULAR SIMULATIONS
}

\author{
Huaichen Zhang, ${ }^{1, *}$ Silvia V. Nedea, ${ }^{1}$ Camilo C. M. Rindt, ${ }^{1}$ Herbert A. Zondag, ${ }^{1}$ David M. J. \\ Smeulders ${ }^{1}$ \\ ${ }^{1}$ Eindhoven University of Technology, Den Dolech 2, Eindhoven, 5612AZ, the Netherlands
}

\begin{abstract}
Sugar alcohols have been recently under investigation for their use as phase change materials in long-term heat storage systems. The thermal performances in such systems are strongly dominated by the nucleation and crystal growth kinetics, which on their turn are linked to the crystal-melt interfacial free energy $\gamma_{\mathrm{SL}}$. We report a novel technique using first principle calculations to accurately predict $\gamma_{\mathrm{SL}}$ of xylitol and $\beta$-Dmannitol, as well as their anisotropy, from molecular modeling. The molecular model is based on a wellproven generalized AMBER force field, which can reliably reproduce the physics during the phase change. The calculation technique is generalized from a cleaving method which has been successfully implemented in monoatomic and rigid polyatomic molecular systems. This method essentially creates an artificial interface and gradually puts the crystal and melt phases into contact under the guidance of a cleaving potential. We extended this method for the calculation of flexible polyatomic molecules. A specific cleaving potential is designed for molecular systems with many degrees of freedoms. We made many efforts to achieve reliable equilibrium Boltzmann sampling and reduction of hysteresis, including the selection of transition paths. The samples of transition states are processed based on the Bennett Acceptance Ratio method. The predicted free energies agree with available experimental estimations, and the large anisotropy in the interfacial free energy is found which could be responsible for the dendritic growth of xylitol and D-mannitol systems as observed in experiments.
\end{abstract}

KEY WORDS: statistical thermodynamics, molecular modeling, interfacial free energy, thermodynamic integration, phase transition, cleaving method

\section{INTRODUCTION}

Phase change materials are extensively used in many industrial and residential applications. One such application involves storing solar heat in summer in order to cut down the energy consumption in the built environment in winter. Recent studies suggest using sugar alcohols (C4-C6 polyols) as seasonal heat storage media [1]. These materials have relatively high latent heat, proper melting temperatures $\left(80-120^{\circ} \mathrm{C}\right)$ for residential heating, and an evident subcooling effect to achieve storage of subcooled liquid at ambient temperature. However, the low nucleation rate and unpredictable growth pattern hinder the heat transfer process, resulting in low and poorly controlled discharge power, which in turn introduces difficulties in large-scale commercial applications. These difficulties make it indispensable to have an in-depth understanding of the nucleation and crystal growth processes.

*Corresponding Huaichen Zhang: h.zhang@tue.nl 
To our knowledge, the key thermodynamic quantity that governs homogeneous nucleation and the morphology of dendritic growth is the crystal-melt interfacial free energy (IFE), $\gamma_{\mathrm{SL}}$. Classical nucleation theory takes an isotropic $\gamma_{\mathrm{SL}}$ as an approximation, but it fails to predict the intricate dendritic growth of sugar alcohol systems as observed in experiments. As a matter of fact, the complicated growth patterns and the morphology therein, are dependent on and very sensitive to the anisotropy of $\gamma_{\mathrm{SL}}$ values. Therefore, accurate anisotropic values of $\gamma_{\mathrm{SL}}$, either from experimental measurements or from theoretical predictions, are of great importance. Many experimental techniques have been developed over the recent years; however, no method is both reliable and easy to carry out [2]. Meanwhile, various simulation techniques have been proposed. These methods include: the cleaving method [3], the capillary fluctuation method (CF) [4], the superheating-undercooling hysteresis method (SUH) [5], the classical nucleation theory based method (CNT-based) [6], the grain boundary mutation method (GBM) [7], the mean lifetime method [8], the metadynamics based method [2], and contact angle simulations (such as [9]). Most of these methods give reliable values of $\gamma_{\mathrm{SL}}$ for the hard sphere or the LennardJones (LJ) models and are claimed by their authors to be transferrable to poly-atomic molecules. However, so far, only a few applications to poly-atomic molecules are found in the literature [10-14], and not all of them are capable of calculating anisotropic values. Additionally, sugar alcohols systems suffer from low nucleation rates and poor crystal growth kinetics. Therefore, simulating spontaneous nucleation using a large box of subcooled metastable liquid is very inefficient considering the length and time scale of molecular simulations, and so are the methods that rely on observable phase boundary transformations (such as CF, CNT-based, GBM). All the above considerations have led us to the relatively mature cleaving method, which is direct, fast, from the fundamentals, and independent of spontaneous phase boundary transformations.

Despite of the successful applications of the cleaving method to LJ and water systems, the extension to flexible poly-atomic organic molecules is not an easy task. On the one hand, sugar alcohol molecules have many more degrees of freedom and require more complicated cleaving potential to align the molecules. On the other hand, bigger molecules have intrinsic hysteresis that require additional treatments. To tackle these problems, we have designed a cleaving potential that is both competent of cleaving and easy to implement. This cleaving potential is readily generalizable to all sugar alcohols or carbon chain molecules. The irreversibility is inherently much stronger in these molecular systems, and thus we made many efforts to reduce this irreversibility. The crystal facet selected for constructing interfacial systems are the ones with the lowest Miller indices. Our calculations are based on a truncated model, with the electrostatic interaction switched off at certain $r_{\mathrm{c}}$. This model is much faster to compute given the long-range electrostatic contributions are negligible. Our results are compared with the much simpler SUH method [5], with discrepancies pointed out and discussed. Finally, the calculation results are used to interpret some of the experimental findings such as the long prismatic-shaped growth of $\beta$-D-mannitol crystal.

\section{METHODOLOGY}

\subsection{Molecular model}

In this case study, the calculations are performed for pure xylitol and D-mannitol crystal-melt interfacial systems, two of the most promising sugar alcohols for seasonal heat storage applications. To choose an appropriate force field, we first considered our scopes and perspectives of the calculations. Since there are no chemical reactions during the phase change, it is possible to use faster non-reactive classical force fields (such as AMBER [15], CHARMM [16] and OPLS-AA [17]) to represent these molecular compounds. These classical models have good performance in non-reactive situations, and are simple to support nanosecond or microsecond scale simulations. This feature is essential to study phase change phenomena, which are generally considered as rare events in molecular simulations. We apply a generalized AMBER force field (gaff) [18] in our molecular models. In this model, every atom is modeled explicitly. The potential energy can be expressed as

$$
E_{p}=E_{\text {bond }}+E_{\text {angle }}+E_{\text {dih }}+E_{\mathrm{LJ}}+E_{\text {el }}
$$


where the energy terms represent total potential energy, bond stretching energy, angle bending energy, dihedral rotation energy, van der Waals interaction with Lennard-Jones form, and electrostatic interaction, respectively, with

$$
\begin{gathered}
E_{\mathrm{bond}}=\sum_{\text {bond }} \frac{1}{4} k_{\mathrm{b}, i j}\left(r_{i j}-r_{0, i j}\right)^{2} \\
E_{\text {angle }}=\sum_{\text {angle }} \frac{1}{2} k_{\mathrm{a}, i j k}\left(\theta_{i j k}-\theta_{0, i j k}\right)^{2} \\
E_{\text {dih }}=\sum_{\text {dihedral }} \sum_{n=0}^{4} \frac{V_{n, i j k l}}{2}\left[1+\cos \left(n \phi_{n, i j k l}-\gamma_{n, i j k l}\right)\right] \\
E_{\mathrm{LJ}}=\sum_{r_{i j}<r_{\mathrm{c}, \mathrm{LJ}}}\left(\frac{A_{i j}}{r_{i j}^{12}}-\frac{B_{i j}}{r_{i j}^{6}}\right) \\
E_{\mathrm{el}}=\sum_{r_{i j}<r_{\mathrm{c}, \mathrm{el}}} \frac{q_{i} q_{j}}{4 \pi \epsilon_{0}}\left(\frac{1}{r_{i j}}-\frac{5}{3 r_{\mathrm{c}, \mathrm{el}}}+\frac{5 r_{i j}^{3}}{3 r_{\mathrm{c}, \mathrm{el}}^{4}}-\frac{r_{i j}^{4}}{r_{\mathrm{c}, \mathrm{el}}^{5}}\right)
\end{gathered}
$$

The parameters $k_{b}, b_{0}, k_{a}, \theta_{0}, V_{n}, \gamma, A, B$, and $q$ are fitted from density functional theory calculations. Details can be found in [18]. $r_{\mathrm{c}, \mathrm{LJ}}, r_{\mathrm{c}, \mathrm{el}}$ are the cut-off radii of LJ interactions and electrostatic interactions. For LJ interactions, Lorentz-Bertelot combination rule is applied in generating non-bonded LJ parameters. To increase calculation speed, the electrostatic interactions are gradually switched off at $r_{c, \text { el }}$ using equation (2e). This switching scheme is in conjunction with a Poisson solver for long range part [19].

The molecular dynamics simulations in this work follows Newtons equations of motions. First, the atomic forces are derived from the negative gradient of $E_{\mathrm{p}}$; then the velocities and displacements are updated using a leap-frog integration scheme in each iteration. More details are given in section 2.4.

\subsection{Free energy calculation}

The free energy difference $\Delta F$ between two states, for example, $\mathrm{A}$ and $\mathrm{B}$, is equivalent to the reversible work needed to transform from one state to the other. Using the definition of the Helmholtz free energy in statistical mechanics, one can design a set of intermediate states by linearly interpolating between the two end states,

$$
E(\lambda)=E_{\mathrm{A}}+\lambda\left(E_{\mathrm{B}}-E_{\mathrm{A}}\right)
$$

with $0 \leq \lambda \leq 1$. Then $\Delta F$ can be obtained by integral over the transition path.

$$
\Delta F=\int_{0}^{1} \frac{\partial F(\lambda)}{\partial \lambda} d \lambda=\int_{0}^{1}\left\langle\frac{\partial E(\lambda)}{\partial \lambda}\right\rangle_{\lambda} d \lambda
$$

where \langle\rangle$_{\lambda}$ denotes ensemble averages. This method is called thermodynamic integration. The derivation is stated elsewhere [20].

The integral in equation (4) can be done numerically by varying $\lambda$ from 0 to 1 in a time-dependent manner. The ensemble average is replaced by a time-dependent term,

$$
\frac{\partial F(\lambda)}{\partial \lambda} d \lambda=\left\langle\frac{\partial E(\lambda)}{\partial \lambda}\right\rangle_{\lambda} d \lambda \simeq \frac{\partial E(\Gamma(t), \lambda)}{\partial \lambda} \dot{\lambda}(t) d t
$$

where $\Gamma(t)$ is a phase space trajectory. Usually the transition is made very slow, because the equal sign is only valid in the limit of infinite time and infinite samples. Therefore, the method is named 'slow growth'. It has been shown that slow growth by a single simulation has intrinsic biases and yields poor results [21]. 
A better method recommended by Shirts and Pande [21] is the Bennett acceptance ratio method (BAR) [22]. The idea is to use the distribution of forward works $W_{\mathrm{F}}$ and reverse works $W_{\mathrm{R}}$ to get the estimator of reversible work with minimal moment bias. However, when the phase spaces between the two end states have little overlap, the estimator yields to large variances. Davidchack et al. [13] have proved that BAR can be used in combination with slow growth by performing multiple forward $(\lambda: 0 \rightarrow 1)$ and reverse $(\lambda: 1 \rightarrow 0)$ simulations. BAR results in an asymptotically unbiased estimator $W$ when both forward and reverse information are available. This estimator $W$ is solved from the following equation:

$$
\sum_{i=1}^{n} \frac{1}{1+\exp \left[\beta\left(W_{\mathrm{F}, i}-W\right)\right]}=\sum_{j=1}^{n} \frac{1}{1+\exp \left[\beta\left(W-W_{\mathrm{R}, j}\right)\right]}
$$

where $\beta=1 / k_{\mathrm{B}} T, k_{\mathrm{B}}$ is the Boltzmann constant, and $T$ is temperature [K]. It is not hard observe the monotonicity on both sides of equation (6). Thus $W$ can be quickly solved numerically. $W_{\mathrm{F}}$ and $W_{\mathrm{R}}$ are defined as

$$
\begin{aligned}
& W_{F}=\left\langle E\left(\lambda_{\mathrm{B}}\right)-E\left(\lambda_{\mathrm{A}}\right)\right\rangle_{\lambda_{\mathrm{A}}} \\
& W_{R}=\left\langle E\left(\lambda_{\mathrm{B}}\right)-E\left(\lambda_{\mathrm{A}}\right)\right\rangle_{\lambda_{\mathrm{B}}}
\end{aligned}
$$

In the work of Davidchack et al., the above definition is replaced by

$$
\begin{gathered}
W_{F}=\int_{0}^{1} \frac{\partial E\left(\Gamma_{\mathrm{F}}(t), \lambda_{\mathrm{F}}\right)}{\partial \lambda_{\mathrm{F}}} \dot{\lambda}_{\mathrm{F}}(t) d \lambda_{\mathrm{F}} \\
W_{R}=\int_{1}^{0} \frac{\partial E\left(\Gamma_{\mathrm{R}}(t), \lambda_{\mathrm{R}}\right)}{\partial \lambda_{\mathrm{R}}} \dot{\lambda}_{\mathrm{R}}(t) d \lambda_{\mathrm{R}}
\end{gathered}
$$

with the integrands taken the from of equation (5). In the limit of infinite forward and reverse measurements, $\Delta F=W$.

Sometimes it is helpful to design benchmark intermediate states $\left(\mathrm{P}_{1}, \mathrm{P}_{2}, \cdots, \mathrm{P}_{N}\right)$ to assist the reversible transition between end states when a direct end-to-end state transition is not feasible. The total free energy difference between the initial and the end states $\Delta F(\mathrm{~A} \rightarrow \mathrm{B})$ is then expressed as the sum of the free energy differences between each pair of adjacent states $\Delta F\left(\mathrm{~A} \rightarrow \mathrm{P}_{1}\right)+\Delta F\left(\mathrm{P}_{1} \rightarrow \mathrm{P}_{2}\right)+\cdots \Delta F\left(\mathrm{P}_{N} \rightarrow \mathrm{B}\right)$. The cleaving method is one such example.

\subsection{Cleaving method}

Developed by Davidchack and Laird in 2000 [3], this method has gone through ten years of development $[12,13,23-26]$. The cleaving potential has evolved from the initial 'pushing wall' into the most recent guiding potential wells in accordance with the crystalline structure. Nevertheless, the concept of cleaving and the four step approach has never changed. These four steps are namely 1. Cleaving solid; 2 . Cleaving liquid; 3. Rearrange boundary conditions; and 4 . Removing cleaving potential. The $\lambda$-dependent potential energy of each step is defined as

$$
\begin{gathered}
E_{1}(\lambda)=E_{\mathrm{S} 1}+E_{\mathrm{S} 1-\mathrm{S} 2}+E_{\mathrm{S} 2}+\lambda \Phi \\
E_{2}(\lambda)=E_{\mathrm{L} 1}+E_{\mathrm{L} 1-\mathrm{L} 2}+E_{\mathrm{L} 2}+\lambda \Phi \\
2 E_{3}(\lambda)=(1-\lambda)\left(E_{\mathrm{S} 1-\mathrm{S} 2}+E_{\mathrm{L} 1-\mathrm{L} 2}\right)+\lambda\left(E_{\mathrm{S} 1-\mathrm{L} 2}+E_{\mathrm{S} 2-\mathrm{L} 1}+E_{\mathrm{S} 1}+E_{\mathrm{S} 2}+E_{\mathrm{L} 1}+E_{\mathrm{L} 2}+2 \Phi\right. \\
E_{4}(\lambda)=E_{\mathrm{S} 1}+E_{\mathrm{S} 1-\mathrm{L} 2}+E_{\mathrm{L} 2}+(1-\lambda) \Phi
\end{gathered}
$$

respectively, where $\Phi$ represent the cleaving potential. S and L denote crystal (solid) and melt (liquid) phases. $E_{\mathrm{S} 1}, E_{\mathrm{S} 2}, E_{\mathrm{L} 1}$, and $E_{\mathrm{L} 1}$ represent the potential energy within each region; while $E_{\mathrm{S} 1-\mathrm{S} 2}, E_{\mathrm{S} 1-\mathrm{L} 2}, E_{\mathrm{L} 1-\mathrm{S} 2}$, and $E_{\mathrm{L} 1-\mathrm{L} 2}$ represent cross-boundary terms. 
(a) Crystal (solid) with cleaving potential

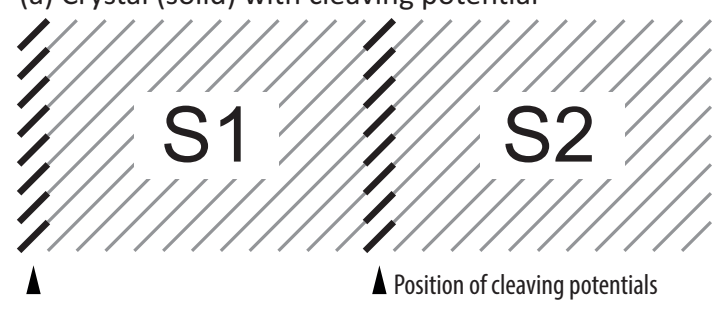

(b) Melt (liquid) with cleaving potential

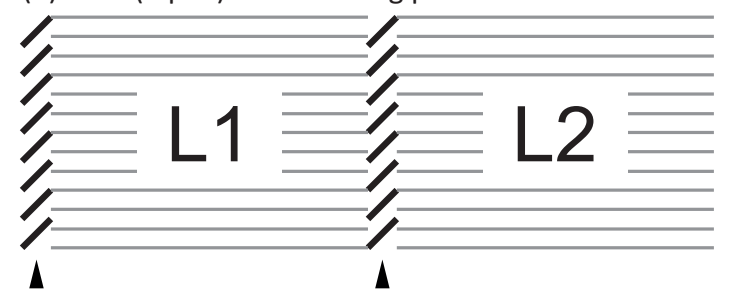

(c) Superposed liquid and solid in pbc rearrangement

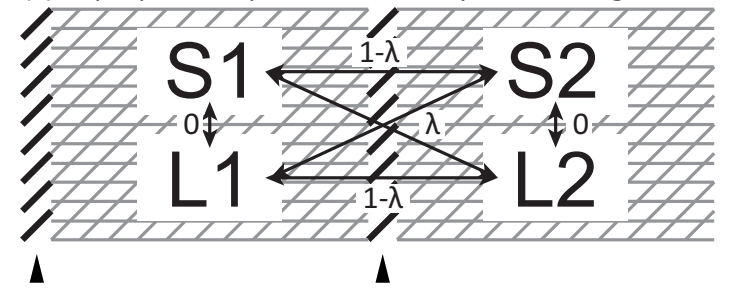

Fig. 1 Graphical illustration of the cleaving method.(a) Duplicated solid with cleaving potential; (b) Duplicated liquid with cleaving potential and a thin crystal layer aligned on the potential; (c) Superposed configuration with specifically defined interaction rules.

In the first two cleaving steps, the cleaving potential is gradually introduced into the system. During this process, some molecules near or at the well sites are gradually trapped in and form a thin crystalline layer. In the third step, the solid-solid and liquid-liquid interactions are gradually switched off, with the solid-liquid interaction switching on simultaneously. This is done by first, duplicating the liquid and solid to form structure shown in figure 1a,1b. Then the solid and liquid systems are spatially superposed. To superpose the solid with the liquid, it requires the solid and the liquid have the same volume and density. The free energy difference between the high density liquid and normal density liquid can be easily evaluated by the work of expansion $\left(\int p \mathrm{~d} V\right)$ and will be discussed in the result section. Finally the interaction strengths are defined based on molecule groups (figure 1c). For example, molecule group L1 and S1 never interact while L1 and S2 can interact with a prefactor $\lambda$. When $\lambda=0$, the system is equivalent to two separate systems of liquid and solid, although they are spatially superposed. When $\lambda$ is turning on from 0 to 1 , the cross-boundary L1-L2 interaction becomes weaker, in contrast to the L1-S2 interaction, and finally, L1 does not 'feel' L2 anymore and instead, interacts only with S2. In this way, the boundary condition is rearranged such that the solid and liquid gradually join together. The molecule groups $\left(S_{i}\right.$ and $\left.L_{i}\right)$ are easy to define, because the pre-formed crystalline structures forbid molecular movement across the boundaries. In step 4, the cleaving potential is switched off. All the above transitions are performed with $\lambda$ switched forwardly $(\lambda: 0 \rightarrow 1)$ and reversely $(\lambda: 1 \rightarrow 0)$ multiple times.

\subsection{Simulation details}

$\beta$-D-mannitol is selected out of all three polymorphs because of its stability and dominance in crystallization studies $[27,28]$. The crystalline structures of xylitol and $\beta$-D-mannitol are obtained from literature [29, 30]

To begin with, the facets with the lowest Miller index, i.e. the (100), (010), and (001) surfaces, are chosen for anisotropic $\gamma_{\mathrm{SL}}$ calculation. The initial configuration of crystalline xylitol consists of $6 \times 4 \times 4,4 \times 6 \times 4,4$ $\times 4 \times 6$ lattices, respectively, each of which contains 4 xylitol molecules (384 molecules in total). The casedependent choice is to ensure a longer box side length in the direction perpendicular to the cleaving surfaces. It increases the distance between two adjacent cleaving walls (coming from periodic boundary condition) and therefore minimizes finite-size effects. For $\beta$-D-mannitol, the initial configuration of solid consists of $9 \times 4 \times$ $2,6 \times 6 \times 2$, and $6 \times 4 \times 3$ lattices, each of which contains 4 D-mannitol molecules. The initial configurations of liquid before cleaving are generated by first heating up the solids far above their melting point. The resultant liquid state can be easily recognized by a sudden increase in the mean squared displacement. Then the melt 
is equilibrated and cooled down gradually. All $\mathrm{C}-\mathrm{H}$ bond and $\mathrm{O}-\mathrm{H}$ bond lengths are constrained using the P-LINCS algorithm [31]. With the constraints, The time step per iteration can be safely set to a relatively large 2 femtosecond. The temperatures for cleaving simulations are set according to the experimental melting conditions, 367.5K for xylitol [32] and 438.0K for D-mannitol [28]. The temperatures are controlled by a Berendsen thermostat [33] with a time constant $0.1 \mathrm{ps}$. All cleaving simulations are done in the constant volume scheme (canonical ensemble). The density for xylitol is $1500.00 \mathrm{~kg} / \mathrm{m}^{3}$, and the density for beta-D-mannitol is $1461.41 \mathrm{~kg} / \mathrm{m}^{3}$.

All simulations are performed using the GROMACS 4.5.5 package [34]. For each transition, the simulation goes for 3 to 5 cycles with 2 million iterations (4ns) in each cycle. The cut-off radii $r_{\mathrm{c}, \mathrm{LJ}}$ and $r_{\mathrm{c}, \mathrm{el}}$ are both set to $1.5 \mathrm{~nm}$.

\section{RESULTS AND DISCUSSIONS}

\subsection{Designing the cleaving potential}

The design of the cleaving potential is crucial to the whole cleaving concept. Here, two criteria should be met: first, after cleaving the liquid (step 2b), the liquid should form a thin crystalline layer; second, the cleaving potential should help achieve reversibility. In addition, the cleaving potential should be easy to implement in available molecular simulation packages without compromising computation speed.

The cleaving potential chosen here has the same form as used by Handel et al. [12]. In his application with water, the orientation and location of a water molecule can be determined and fixed by fixing the coordinate of the oxygen atom and a well-defined orientation vector. In this work, the polyatomic molecules are much more complicated. For example, xylitol $\left(\mathrm{C}_{5} \mathrm{H}_{12} \mathrm{O}_{5}\right)$ has 22 atoms and 66 degrees of freedom (DOF), in contrast with the rigid water model which has only 6 DOF. Defining the orientation of the xylitol molecule requires 21 independent vectors. Besides, the orientation itself in the crystalline structure has four possibilities $\left(\mathrm{P} 2{ }_{1} 2_{1} 2_{1}\right.$ space group, orthorhombic), making it necessary to define orientations in a location dependent manner.

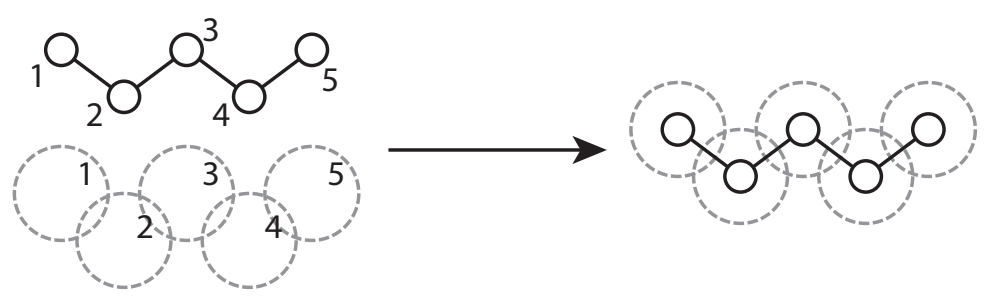

(a)

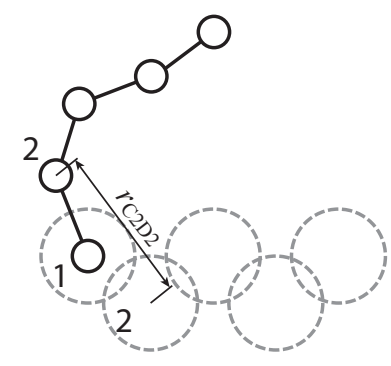

(c) (b)

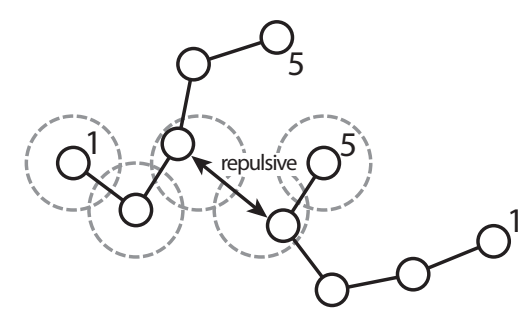

(d)

Fig. 2 Schematic diagram of a molecule aligned by the cleaving potential. Small solid circles represent carbon atoms in a xylitol molecule; big dashed circles are potential wells. Only atoms and wells with the same index number can interact with each other. (a) before alignment; (b) after alignment; (c) atom by atom alignment; (d) two-molecule-in-one-chain situation. 
To avoid these complications, we decided to control the orientation of the molecules by only considering the carbon chain atoms. This is done as follows. Take xylitol (001) case as an example. First of all, the configuration of xylitol crystalline structure is generated by duplicating $4 \times 4 \times 6$ lattices (section 2.4). Then the coordinates are scaled to match the density. The generated configuration look similar to the one shown in figure 3a. Secondly, all carbon atoms are labeled as type $\mathrm{C}_{1}$ to $\mathrm{C}_{5}$ according to their relative positions in the carbon chain (figure 2a) (for D-mannitol, $\mathrm{C}_{1}$ to $\mathrm{C}_{6}$, same below). Thirdly, one layer of molecules on the (001) surface is selected. The coordinates of the carbon atoms in the selected molecules are read and saved. Fourthly, a group of dummy atoms is generated according to the coordinates saved in the previous step. These dummy atoms are labeled as type $\mathrm{D}_{1}$ to $\mathrm{D}_{5}$, and they occupy exactly the same spots as the selected carbon atoms, $\mathrm{C}_{1}$ to $\mathrm{C}_{5}$, respectively. These dummy atoms will be fixed throughout the cleaving simulations. At last, the topology is defined such that the atoms of type $\mathrm{C}_{i}$ only interact with atoms of type $\mathrm{D}_{i}$, with $i=1$ to 5 . The cleaving potential $\Phi$ is then defined as

$$
\Phi=\sum_{i=1}^{5} \sum_{r_{\mathrm{C}_{i} \mathrm{D}_{i}}<r_{\mathrm{c}, \mathrm{w}}} d_{i}\left[\left(r_{\mathrm{C}_{i} \mathrm{D}_{i}} / r_{\mathrm{c}, \mathrm{w}}\right)^{2}-1\right]^{3}
$$

where $d_{i}$ is $i$-dependent potential well depth, $r_{\mathrm{c}, \mathrm{w}}$ is the well width beyond which the interaction is cut off.

The above definition ensures the sugar alcohol molecules to lie on the dummy chains with the correct orientations, as illustrated in figure $2 \mathrm{~b}$. The major advantage of this design is the possibility to treat these potential wells as atoms, and the interactions between these well atoms and the real atoms can be treated as non-bonded interactions, with a user defined potential. This enables the use of many commercial software packages without additional programming.

The well width $r_{\mathrm{c}, \mathrm{w}}$ in equation (10) is $0.3 \mathrm{~nm}$, approximately twice the $\mathrm{C}-\mathrm{C}$ bond lengths. When a molecule is only partially trapped in a potential well chain, like the one in figure $2 \mathrm{c}$, this choice of $r_{\mathrm{c}, \mathrm{w}}$ is sufficient to guide the rest of the molecule to sit in the well chain atom by atom. This is because $r_{\mathrm{C}_{2} \mathrm{D}_{2}}$ never exceeds twice the $\mathrm{C}-\mathrm{C}$ bond length if $r_{\mathrm{C}_{1} \mathrm{D}_{1}}=0$. One can argue that an even larger $r_{\mathrm{c}, \mathrm{w}}$ can better assist to align the molecules. This is true. However, a larger $r_{\mathrm{c}, \mathrm{w}}$ also results in oversized potential wells that can easily trap multiple carbon atoms (of the same index number $i$ ) in one potential well. This situation should apparently be avoided.

Another undesired situation is called 'two-molecule-in-one-chain', as illustrated in figure $2 \mathrm{~d}$. This situation can be avoided by designing $d_{i}$ in an index number $i$-dependent manner. The idea is to make deeper potential wells for the center carbon atoms. In this way, when the center carbon atoms are trapped in the wells, they are dominant enough to assist the rest of their molecules to 'kick' the other molecules away using the LJ repulsive force. The well depths are in the order of $10 \mathrm{~kJ} / \mathrm{mol}$ (the reason will be given in section 3.3), and the individual values are given as follows. In the case of xylitol, $\mathrm{d}_{3}=45 \mathrm{~kJ} / \mathrm{mol}_{1} \mathrm{~d}_{2}=\mathrm{d}_{4}=25 \mathrm{~kJ} / \mathrm{mol}$, and $\mathrm{d}_{1}=\mathrm{d}_{5}=20 \mathrm{~kJ} / \mathrm{mol}$. For D-mannitol, $\mathrm{d}_{3}=\mathrm{d}_{4}=45 \mathrm{~kJ} / \mathrm{mol}, \mathrm{d}_{2}=\mathrm{d}_{5}=25 \mathrm{~kJ} / \mathrm{mol}$, and $\mathrm{d}_{1}=\mathrm{d}_{6}=20 \mathrm{~kJ} / \mathrm{mol}$. The number of cleaving potential well chains $N$ in xylitol case is 32 ( $32 D_{i}$ each, $i=1$ to 5); and in D-mannitol case is 32 for the (100) surface or 24 for the (010) surface and (001) surface.

\subsection{Reducing irreversibility}

As the definition for free energy being the reversible work, the reversibility of the transitions is the most important criterion of choosing benchmark intermediate states and choosing cleaving potential. It is already pointed out by Handel et al. [12] that the purpose of forming a crystalline layer in step 2 is to reduce the irreversibility in step 3, by transferring the irreversibility to step 2 . The irreversibility in step 2 can then be easily be reduced or eliminated at a higher temperature or a reduced potential.

In this work, we adopted the same approach. The liquid potential $E_{\mathrm{L}}$ (defined as $E_{\mathrm{L}}=E_{\mathrm{L} 1}+E_{\mathrm{L} 1-\mathrm{L} 2}+E_{\mathrm{L} 2}$ ) is reduced to $30 \%$. However, this reduction is only applied upon the non-bonded terms $\left(E_{\mathrm{LJ}}\right.$ and $\left.E_{\mathrm{el}}\right)$ and 
the dihedral term $\left(E_{\mathrm{dih}}\right)$. This is to avoid energy equipartition on vibration degrees of freedom that causes unnecessary poor phase space overlap. The potential is scaled back to full strength after the cleaving. In this way, the cleaving step 2 is split into 3 substeps: step $2 a$, step 2 b, and step $2 c$, defined by the following equations, respectively

$$
\begin{gathered}
E_{2 a}(\lambda)=(1-0.7 \lambda)\left(E_{\mathrm{L}, \text { dih }}+E_{\mathrm{L}, \mathrm{LJ}}+E_{\mathrm{L}, \mathrm{el}}\right)+E_{\mathrm{L}, \text { bond }}+E_{\mathrm{L}, \text { angle }} \\
E_{2 b}(\lambda)=0.3\left(E_{\mathrm{L}, \mathrm{dih}}+E_{\mathrm{L}, \mathrm{LJ}}+E_{\mathrm{L}, \mathrm{el}}\right)+E_{\mathrm{L}, \text { bond }}+E_{\mathrm{L}, \text { angle }}+\lambda \Phi \\
E_{2 c}(\lambda)=(0.3+0.7 \lambda)\left(E_{\mathrm{L}, \mathrm{dih}}+E_{\mathrm{L}, \mathrm{LJ}}+E_{\mathrm{L}, \mathrm{el}}\right)+E_{\mathrm{L}, \text { bond }}+E_{\mathrm{L}, \text { angle }}+\lambda \Phi
\end{gathered}
$$

The effect of further reduction of potential on reducing hysteresis is no longer evident. Instead, it will increase the free energy difference in step $2 \mathrm{a}$ and $2 \mathrm{c}$, resulting in larger inaccuracies. The residue hysteresis in step $2 \mathrm{~b}$ at $30 \%$ potential is negligible as illustrated in figure 5 .

\subsection{Performance of the cleaving potential}

Snapshots are taken to examine if the a thin crystal layer is formed at the cleaving potential positions after step $2 b$. One such example is shown in figure $3 \mathrm{~b}$. On the left of $3 \mathrm{~b}$ for the (001) surface of xylitol, the molecules in good structural order are the ones trapped in the cleaving potential wells. To further ascertain that all the potential wells are filled, a histogram of distances (denoted as $l_{i}$ ) of the nearest $\mathrm{C}_{i}$ type atom around each $\mathrm{D}_{i}$ type well is plotted for this snapshot (figure 4). It can be seen that the largest distance between the pairs never exceeds $0.12 \mathrm{~nm}$, much less than the radius of the cleaving potential, $0.3 \mathrm{~nm}$.

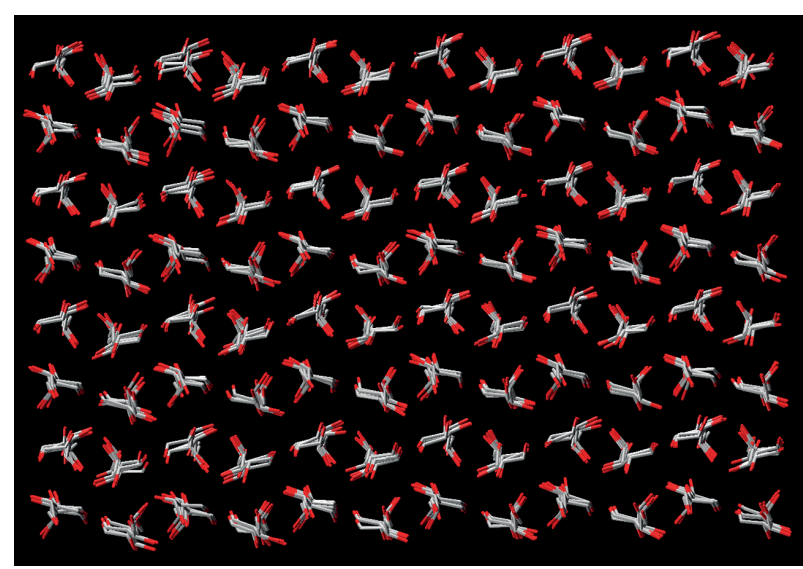

$\triangle$ Position of cleaving potential

(a) Final configuration of step 1

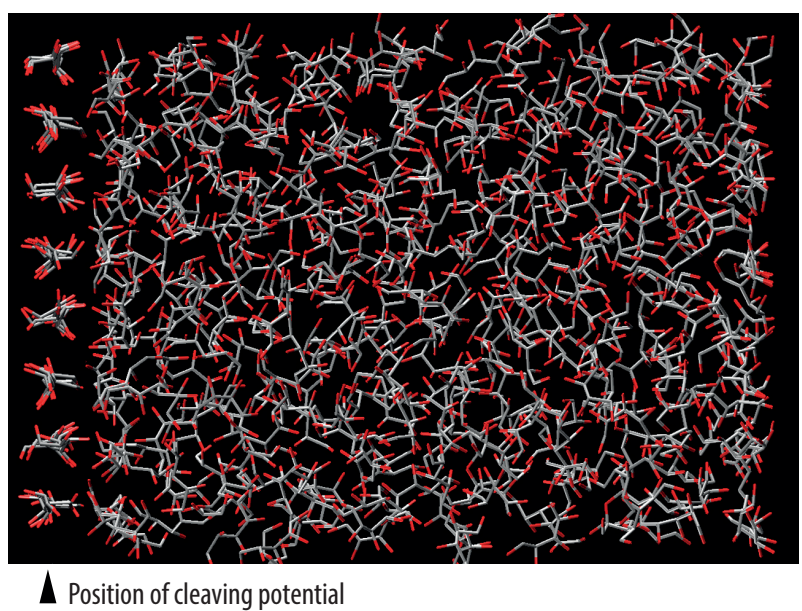

(b) Final configuration of step $2 b$

Fig. 3 Configuration of xylitol liquid after cleaving step 2b. The cleaved surface belong to the (001) surface. A one-molecule layer thick crystalline layer is formed along the cleaving potential. Hydrogen atoms are made invisible.

Three cycles of integrand $\mathrm{d} F / \mathrm{d} \lambda$ for step $2 \mathrm{~b}$ are plotted in figure 5 to examine the hysteresis. The forward and reverse curves almost overlap each other, showing a negligible hysteresis. The shapes of the curves can very well answer the question whether the magnitudes of well depths $d_{i}$ are chosen properly. Define the tails of the curves to be the almost flat part at larger $\lambda$ values (in the case of figure5, at $\lambda>0.8$ ). If the cleaving continues such that $\lambda$ approaches infinity, the tail will converge to a number, which is $-|\max (\Phi)|=-N \sum d_{i}$ (in the xylitol case, $-4320 \mathrm{~kJ} / \mathrm{mol}$ ). If the potential wells are deeper, the atoms will be trapped in in an earlier stage and the tails will become longer. At the same time, the tails will converge to a lower value. Both the above consequences will result in much lower values of $W_{\mathrm{F}}$ and $W_{\mathrm{R}}$ in equation (8) after the integral. And the larger $W_{\mathrm{F}}$ and $W_{\mathrm{R}}$ values will result in a larger free energy difference in step $2 b$, which introduces unnecessary inaccuracies. On the contrary, shallower potential wells produces shorter tails and an insufficiently cleaved surface (molecules have chances to escape), which dissatisfy the first cleaving criterion in section 3.1. 


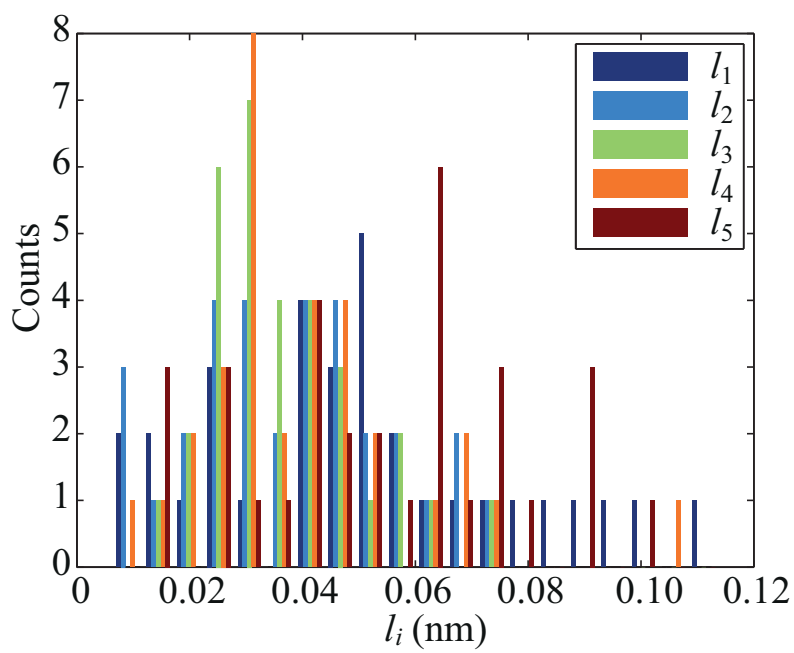

Fig. 4 A histogram of the distances of the nearest $C_{i}$ to each $D_{i}$. The histogram is made according to the configuration in figure 3 .

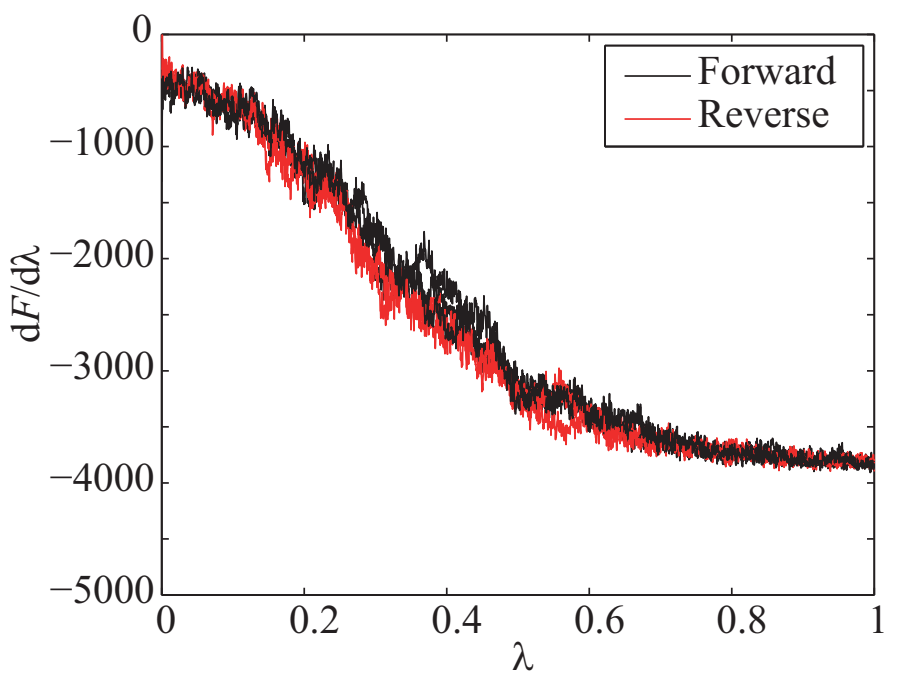

Fig. 5 Integrand $\mathrm{d} F / \mathrm{d} \lambda$ in equation (8) with respect to $\lambda .3$ forward and 3 reverse transition results are shown.

\subsection{Anisotropic interfacial free energy}

The anisotropic IFE values are listed in Table 1. $\Delta F_{i}$ values are estimated by the unbiased estimator $W$ in accordance with equation (6). The subscripts denote each cleaving step. Note that in each crystal-melt coexistent system, there are two interfacial areas. Therefore, the free energy sum of each step should be divided by twice the intersection area, $2 A$.

The anisotropy in both systems are large. Although the same cleaving potential is applied to both solid and liquid, $\Delta F_{1}$ and $\Delta F_{2 \mathrm{~b}}$ values are very different. Because the cleaving potential is tailored according to the atomic arrangement of the crystal, the crystalline structures are easier to trap in the potential wells. The cleaving step $2 \mathrm{a}$ and $2 \mathrm{c}$ have the opposite effect on the system, so are step $1+2 \mathrm{~b}$ and 4 . The sum $\sum \Delta F$ can therefore be viewed as the residue of the cancellation of each terms. This makes $\sum \Delta F$ very sensitive to the individual $\Delta F$ terms. This sensitivity is the reason for the choice of not-too-deep cleaving potential and the $30 \%$ potential scaling argued in both section 3.3 and section 3.2. The free energy difference contributed by the liquid density change is not included. This contribution can be evaluated by the work of expansion or compression. Since both the initial bulk liquid state and the final solid-liquid interfacial system involve high density liquid, the 
Table 1 Anisotropic interfacial free energies $\left({ }^{*} \mathrm{Xylitol},{ }^{\dagger} \mathrm{D}-\mathrm{mannitol}\right)$

\begin{tabular}{|l|cccccc|c|c|c|}
\hline & $\begin{array}{c}\Delta F_{1} \\
(\mathrm{~kJ} / \mathrm{mol})\end{array}$ & $\begin{array}{c}\Delta F_{2 \mathrm{a}} \\
(\mathrm{kJ} / \mathrm{mol})\end{array}$ & $\begin{array}{c}\Delta F_{2 \mathrm{~b}} \\
(\mathrm{~kJ} / \mathrm{mol})\end{array}$ & $\begin{array}{c}\Delta F_{2 \mathrm{c}} \\
(\mathrm{kJ} / \mathrm{mol})\end{array}$ & $\begin{array}{c}\Delta F_{3} \\
(\mathrm{~kJ} / \mathrm{mol})\end{array}$ & $\begin{array}{c}\Delta F_{4} \\
(\mathrm{~kJ} / \mathrm{mol})\end{array}$ & $\begin{array}{c}\sum \Delta F \\
(\mathrm{~kJ} / \mathrm{mol})\end{array}$ & $\begin{array}{c}2 A \\
\left(\mathrm{~nm}^{2}\right)\end{array}$ & $\begin{array}{c}\gamma_{\mathrm{SL}} \\
\left(\mathrm{mJ} / \mathrm{m}^{2}\right)\end{array}$ \\
\hline$(100)^{*}$ & -4050.86 & & -2623.19 & -8389.94 & +85.31 & 7810.54 & 1102.61 & 25.9034 & 70.683 \\
$(010)^{*}$ & -4050.12 & 8313.40 & -2953.95 & -8291.49 & -18.82 & 7647.05 & 655.48 & 23.8518 & 45.634 \\
$(001)^{*}$ & -4069.99 & & -2960.45 & -8257.84 & +315.84 & 8025.19 & 1208.23 & 24.0269 & 83.503 \\
\hline$(100)^{\dagger}$ & -4950.42 & & -3676.55 & -12724.53 & +411.95 & 9888.54 & 1280.61 & 23.6141 & 90.052 \\
$(010)^{\dagger}$ & -3810.48 & 12537.59 & -2788.65 & -12686.54 & +131.11 & 7759.78 & 1077.26 & 22.8631 & 78.241 \\
$(001)^{\dagger}$ & -3987.58 & & -2736.35 & -12633.00 & +127.63 & 7533.87 & 778.35 & 23.3600 & 55.328 \\
\hline
\end{tabular}

total free energy contribution is then the expansion work difference between both systems. Since both liquids follow the same expansion path, the expansion work contribution to a large extent cancel each other, and is hence not included in the calculations.

Overall, the interfacial free energies are larger than those of xylitol. This means more free energy is needed to form a unit area of $\beta$-D-mannitol crystal-melt interface than xylitol.

\subsection{Comparison with the superheating-undercooling hysteresis method}

The superheating-undercooling hysteresis (SUH) method [5] is based on empirical formulae. It offers a way to quickly estimate the isotropic interfacial free energy from a simple set of simulations. Wang et al. [11] applied the SUH method on water systems, and obtained reasonable agreement with experimental and simulation results. Based on the theory of the original paper, $\gamma_{\mathrm{SL}}$ is related to a melting temperature, a volumetric heat of fusion $\Delta H_{\mathrm{m}, \mathrm{V}}^{2}$, and a dimensionless nucleation barrier parameter $\beta$ in the following form [5]

$$
\gamma_{S L}=\left(\frac{3}{16 \pi} \beta k_{\mathrm{B}} T_{\mathrm{m}} \Delta H_{\mathrm{m}, \mathrm{V}}^{2}\right)^{1 / 3}
$$

where $T_{\mathrm{m}}$ is the melting temperature in the limit of a zero heating or cooling rate. $\beta$ can be decided by an empirical formula

$$
\beta=\left(59.4-2.33 \log _{10} Q\right) \frac{T_{+/-}}{T}\left(\frac{T_{+/-}}{T}-1\right)^{2}
$$

where $T_{+/-}$is the melting temperature obtained from SUH simulations (figure 6) or experiments, $Q$ is the scalar heating or cooling rate in $[\mathrm{K} / \mathrm{s}]$.

In our simulations, we chose 3 different scaling heating (or cooling) rates with $Q=1,0.2$, or $0.05 \mathrm{~K} / \mathrm{ps}$. The initial configurations are generated according to their corresponding perfect crystalline structures. Then the systems are heated up with the aforementioned heating rates until a sudden drop in the density profile occurs. Then the systems are cooled down using the same $Q$. The pressures are set to 1 bar and controlled by a Berendsen barostat [33] with a time constant $0.5 \mathrm{ps}$. The maximum superheating temperatures $T_{+}$are determined as the points with the least radii of curvature around the density drop region. In both xylitol and $\beta$-D-mannitol cases (figure 6), the first order phase transitions during the heating-up process can be easily identified from the sudden drop in the density profile. With lower heating rates, the materials melt at lower temperatures, resulting in lower $T_{+}$values. During the cooling process, the crystallization is not observed. Instead, the liquids become glassy.

Table 2 lists the calculation results using the simulation data. $T_{m}$ is the experimental melting temperatures. The results from SUH match those from the cleaving method. In both cases, SUH gives values closer to the lowest value from the cleaving method. This is not surprising given that SUH mimics a spontaneous process which has preferred directions of melting. The system finds for itself the lowest energy barrier before it goes across. 

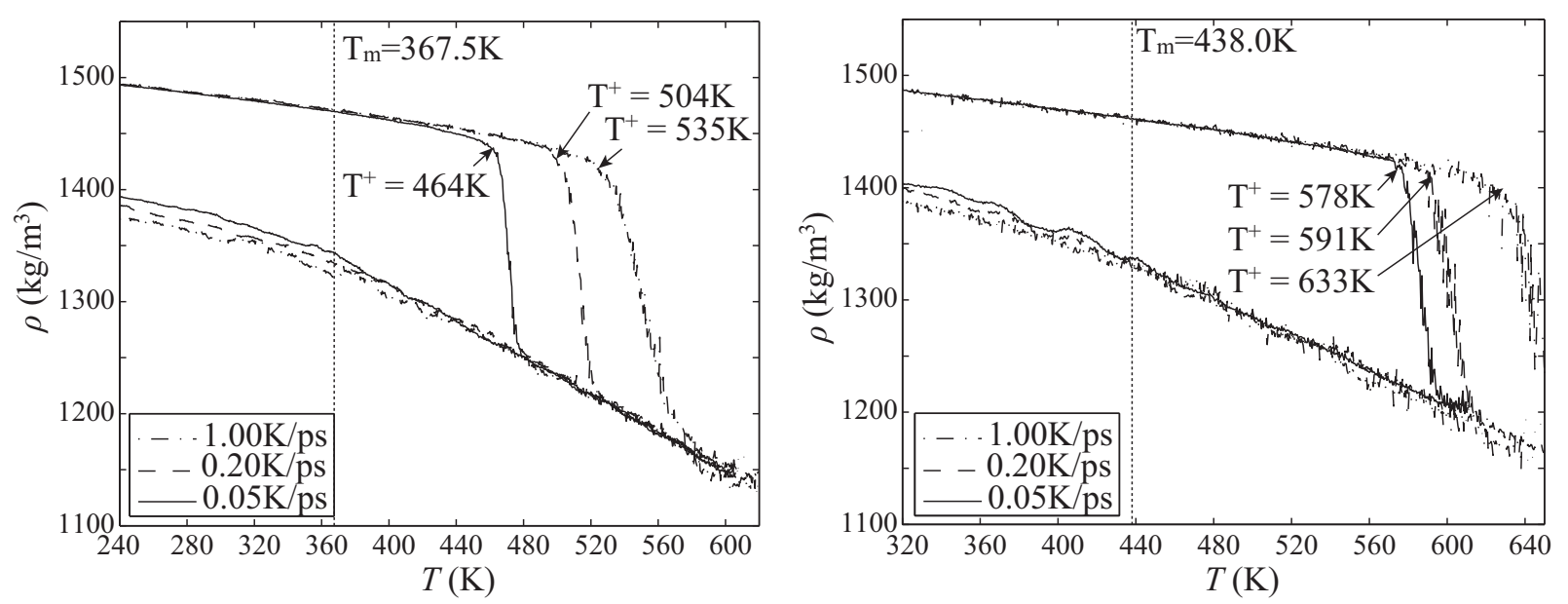

Fig. 6 The density profiles by superheating-undercooling simulations at various heating-up and cooling rates. Left: xylitol; Right: $\beta$-D-mannitol.

Table 2 Isotropic approximations of $\gamma_{\mathrm{SL}}\left(T_{\mathrm{m}}\right.$ and $\Delta H_{\mathrm{m}, \mathrm{V}}$ taken from [35])

\begin{tabular}{|c|c|c|c|c|c|c|c|}
\hline & $Q(\mathrm{~K} / \mathrm{s})$ & $T^{+}(\mathrm{K})$ & $T_{\mathrm{m}, \exp }(\mathrm{K})$ & $\beta$ & $\Delta H_{\mathrm{m}, \mathrm{V}}\left(\mathrm{J} / \mathrm{m}^{3}\right)$ & $\gamma_{\mathrm{SL}}\left(\mathrm{mJ} / \mathrm{m}^{2}\right)$ & $\bar{\gamma}_{\mathrm{SL}}\left(\mathrm{mJ} / \mathrm{m}^{2}\right)$ \\
\hline Xylitol & $1 \times 10^{12}$ & 535 & 367.5 & 9.51 & $2.903 \times 10^{8}$ & 62.4 & \\
Xylitol & $2 \times 10^{11}$ & 504 & 367.5 & 6.26 & $2.903 \times 10^{8}$ & 54.3 & 53.0 \\
Xylitol & $5 \times 10^{10}$ & 464 & 367.5 & 3.00 & $2.903 \times 10^{8}$ & 42.5 & \\
\hline D-mannitol & $1 \times 10^{12}$ & 633 & 438.0 & 9.01 & $4.595 \times 10^{8}$ & 88.2 & \\
D-mannitol & $2 \times 10^{11}$ & 591 & 438.0 & 5.44 & $4.595 \times 10^{8}$ & 74.6 & 77.8 \\
D-mannitol & $5 \times 10^{10}$ & 578 & 438.0 & 4.65 & $4.595 \times 10^{8}$ & 70.8 & \\
\hline
\end{tabular}

\subsection{Other discussions}

It is possible to predict the crystal habit from the anisotropic interfacial free energies via Wulff's construction rules [36]. However, an accurate prediction relies on a detailed calculation involving all potential crystal facets. Nevertheless, the available $\gamma_{\mathrm{SL}}$ values suggest grains with uneven aspect ratios. In recent experiments of homogeneous nucleation and crystal growth in a subcooled liquid, anisotropic long needle shaped prisms are observed to be the main growth pattern of D-mannitol while strong surface nucleation and a dendritic growth pattern dominates the growth of xylitol [35]. Both phenomena can be ascribed to the presence of large anisotropy of $\gamma_{\mathrm{SL}}$.

A simple arithmetic average of anisotopic $\gamma_{\mathrm{SL}}$ values give $\gamma_{\mathrm{SL}, \mathrm{xyl}}=67 \mathrm{~mJ} / \mathrm{m}^{2}$ and $\gamma_{\mathrm{SL}, \mathrm{man}}=75 \mathrm{~mJ} / \mathrm{m}^{2}$. From classical nucleation theory, the average population of critical nuclei $n^{*}$ is proportional to $\exp \left(-\Delta G^{*} / k_{\mathrm{B}} T\right)$, where $\Delta G^{*}=\beta\left(T_{\mathrm{m}} / \Delta T\right)^{2}$ is the free energy barrier, $\beta$ is the dimensionless barrier parameter in equation (12) and $\Delta T$ is the degree of subcooling. Taking the above average $\gamma_{\mathrm{SL}}$ values, we have $\beta_{\mathrm{xyl}}=11.8$, and $\beta_{\operatorname{man}}$ $=5.5$. The free energy barrier at small $\Delta T$ is hence small for D-mannitol compared with that of xylitol at the same $\Delta T$. This agrees with experimental observations, where $\mathrm{D}$-mannitol has a substantial faster kinetics during crystallization [35]. The linear scaling between $\gamma_{\mathrm{SL}}$ and $T_{\mathrm{m}}$ still exists, although this rule was only assigned to metal systems at the first place. This scaling effect also applies to water systems [13]. The validity of the scaling rule opens up the possibility of estimating interfacial free energies of unknown systems. 


\section{CONCLUSIONS}

We have successfully extended and implemented the cleaving method on sugar alcohol systems and calculated some selected anisotropic interfacial free energy values. During the implementation, specific cleaving potentials were designed, to tackle the intrinsic irreversibility to the largest extent. This cleaving potential is easily generalizable to other sugar alcohol systems. The anisotropic values were compared with the superheatingundercooling hysteresis method, and the results showed good agreement. The linear scaling rule of interfacial free energy over melting temperature seems to apply to sugar alcohol systems as well, making future estimations of unknown systems possible. We bridged up the scales to understand some experimental phenomena extrapolated from the fundamentals. We found that the large anisotropies in the interfacial free energies could be responsible for the dendritic growth of xylitol and the needle shaped prismatic growth of D-mannitol.

\section{ACKNOWLEDGMENTS}

The research leading to these results has received funding from the European Community's Seventh Framework Programme (FP7/2007-2013) under grant agreement 296006.

\section{REFERENCES}

[1] Elena Palomo del Barrio. SAM.SSA: Sugar alcohol based materials for seasonal storage applications. @Website: http://samssa.eu/, 2013.

[2] Stefano Angioletti-Uberti, Michele Ceriotti, Peter D. Lee, and Mike W. Finnis. Solid-liquid interface free energy through metadynamics simulations. Phys. Rev. B, 81:125416, Mar 2010.

[3] Ruslan L. Davidchack and Brian B. Laird. Direct calculation of the hard-sphere crystal/melt interfacial free energy. Phys. Rev. Lett., 85:4751-4754, Nov 2000.

[4] J. J. Hoyt, Mark Asta, and Alain Karma. Method for computing the anisotropy of the solid-liquid interfacial free energy. Phys. Rev. Lett., 86:5530-5533, Jun 2001.

[5] Sheng-Nian Luo, Thomas J. Ahrens, Tahir Çă̆ in, Alejandro Strachan, William A. Goddard, and Damian C. Swift. Maximum superheating and undercooling: Systematics, molecular dynamics simulations, and dynamic experiments. Phys. Rev. B, 68:134206, Oct 2003.

[6] Xian-Ming Bai and Mo Li. Calculation of solid-liquid interfacial free energy: A classical nucleation theory based approach. The Journal of Chemical Physics, 124(12):-, 2006.

[7] I. Toda-Caraballo, P.D. Bristowe, and C. Capdevila. A molecular dynamics study of grain boundary free energies, migration mechanisms and mobilities in a bcc fe-20cr alloy. Acta Materialia, 60(3):1116 - 1128, 2012.

[8] Vladimir G. Baidakov and Azat O. Tipeev. Crystal nucleation and the solidliquid interfacial free energy. The Journal of Chemical Physics, 136(7):-, 2012.

[9] Bo Shi and Vijay K. Dhir. Molecular dynamics simulation of the contact angle of liquids on solid surfaces. The Journal of Chemical Physics, 130(3):-, 2009.

[10] Xiaobing Feng and Brian B. Laird. Calculation of the crystal-melt interfacial free energy of succinonitrile from molecular simulation. The Journal of Chemical Physics, 124(4):-, 2006.

[11] Jun Wang, Yuk Wai Tang, and X. C. Zeng. Solidliquid interfacial free energy of water: a molecular dynamics simulation study. Journal of Chemical Theory and Computation, 3(4):1494-1498, 2007.

[12] Richard Handel, Ruslan L. Davidchack, Jamshed Anwar, and Andrey Brukhno. Direct calculation of solid-liquid interfacial free energy for molecular systems: Tip4p ice-water interface. Phys. Rev. Lett., 100:036104, Jan 2008.

[13] Ruslan L. Davidchack, Richard Handel, Jamshed Anwar, and Andrey V. Brukhno. Ice ihwater interfacial free energy of simple water models with full electrostatic interactions. Journal of Chemical Theory and Computation, 8(7):2383-2390, 2012.

[14] E. Sanz, C. Vega, J. R. Espinosa, R. Caballero-Bernal, J. L. F. Abascal, and C. Valeriani. Homogeneous ice nucleation at moderate supercooling from molecular simulation. Journal of the American Chemical Society, 135(40):15008-15017, 2013. 
[15] Wendy D. Cornell, Piotr Cieplak, Christopher I. Bayly, Ian R. Gould, Kenneth M. Merz, David M. Ferguson, David C. Spellmeyer, Thomas Fox, James W. Caldwell, and Peter A. Kollman. A second generation force field for the simulation of proteins, nucleic acids, and organic molecules. Journal of the American Chemical Society, 117(19):5179-5197, 1995.

[16] A. D. MacKerell, D. Bashford, Bellott, R. L. Dunbrack, J. D. Evanseck, M. J. Field, S. Fischer, J. Gao, H. Guo, S. Ha, D. Joseph-McCarthy, L. Kuchnir, K. Kuczera, F. T. K. Lau, C. Mattos, S. Michnick, T. Ngo, D. T. Nguyen, B. Prodhom, W. E. Reiher, B. Roux, M. Schlenkrich, J. C. Smith, R. Stote, J. Straub, M. Watanabe, J. WirkiewiczKuczera, D. Yin, and M. Karplus. All-atom empirical potential for molecular modeling and dynamics studies of proteins. The Journal of Physical Chemistry B, 102(18):3586-3616, 1998.

[17] William L. Jorgensen, David S. Maxwell, and Julian Tirado-Rives. Development and testing of the opls all-atom force field on conformational energetics and properties of organic liquids. Journal of the American Chemical Society, 118(45):11225-11236, 1996.

[18] Junmei Wang, Romain M. Wolf, James W. Caldwell, Peter A. Kollman, and David A. Case. Development and testing of a general amber force field. Journal of Computational Chemistry, 25(9):1157-1174, 2004.

[19] W.F. van Gunsteren, P.K. Weiner, and A.J. Wilkinson. Computer Simulation of Biomolecular Systems: Theoretical and Experimental Applications. Computer Simulations of Biomolecular Systems. Springer, 2010.

[20] D. Frenkel and B. Smit. Understanding Molecular Simulation: From Algorithms to Applications. Computational science series. Elsevier Science, 2001.

[21] Michael R. Shirts and Vijay S. Pande. Comparison of efficiency and bias of free energies computed by exponential averaging, the bennett acceptance ratio, and thermodynamic integration. The Journal of Chemical Physics, 122(14):-, 2005.

[22] Charles H Bennett. Efficient estimation of free energy differences from monte carlo data. Journal of Computational Physics, 22(2):245 - 268, 1976.

[23] R L Davidchack and B B Laird. Direct calculation of the crystal-melt interfacial free energies for continuous potentials: Application to the lennard-jones system. Journal of Computational Physics, 118:7651-7657, apr 2003.

[24] Brian B. Laird and Ruslan L. Davidchack. Direct calculation of the crystalmelt interfacial free energy via molecular dynamics computer simulation. The Journal of Physical Chemistry B, 109(38):17802-17812, 2005. PMID: 16853283.

[25] Ruslan L. Davidchack. Hard spheres revisited: Accurate calculation of the solidliquid interfacial free energy. The Journal of Chemical Physics, 133(23):-, 2010.

[26] Yan Mu and Xueyu Song. Calculations of crystal-melt interfacial free energies by nonequilibrium work measurements. The Journal of Chemical Physics, 124(3):-, 2006.

[27] RaghuK. Cavatur, N.Murti Vemuri, Abira Pyne, Zofia Chrzan, David Toledo-Velasquez, and Raj Suryanarayanan. Crystallization behavior of mannitol in frozen aqueous solutions. Pharmaceutical Research, 19(6):894-900, 2002.

[28] Peng Ye and Thomas Byron. Characterization of d-mannitol by thermal analysis, ftir, and raman spectroscopy. American laboratory, 40(14):24-27, 2008.

[29] Anders Østergaard Madsen, Sax Mason, and Sine Larsen. A neutron diffraction study of xylitol: derivation of mean square internal vibrations for $\mathrm{H}$ atoms from a rigid-body description. Acta Crystallographica Section B, 59(5):653-663, Oct 2003.

[30] Frank R. Fronczek, Haidy Nasr Kamel, and Marc Slattery. Three polymorphs ( $\alpha$, $\beta$, and $\delta)$ of D-mannitol at $100 \mathrm{~K}$. Acta Crystallographica Section C, 59(10):0567-o570, Oct 2003.

[31] Berk Hess. P-lincs: A parallel linear constraint solver for molecular simulation. Journal of Chemical Theory and Computation, 4(1):116-122, 2008.

[32] Bo Tong, Zhi-Cheng Tan, Quan Shi, Yan-Sheng Li, Dan-Ting Yue, and Shao-Xu Wang. Thermodynamic investigation of several natural polyols (i): Heat capacities and thermodynamic properties of xylitol. Thermochimica Acta, 457(12):20 - 26, 2007.

[33] Herman JC Berendsen, J P1 M Postma, Wilfred F van Gunsteren, ARHJ DiNola, and JR Haak. Molecular dynamics with coupling to an external bath. The Journal of chemical physics, 81:3684, 1984.

[34] B. Hess, C. Kutzner, D. van der Spoel, and E. Lindahl. GROMACS 4: Algorithms for Highly Efficient, LoadBalanced, and Scalable Molecular Simulation. J. Chem. Theory Comput., 4(3):435-447, February 2008. 
[35] Roel M. J. van Wissen. Phase change behavior of sugar alcohol based heat storage materials. Master's thesis, Eindhoven University of Technology, 072013.

[36] R. Cerf and J. Picard. The Wulff Crystal in Ising and Percolation Models: Ecole D'Etâe de Probabilitâes de Saint-Flour XXXIV, 2004. Number no. 1878 in Ecole d'Ete de Probabilitès de St. Flour. 2006. 